\title{
A Location-Adaptive Human-Centered Audio Email Notification Service for Multi-user Environments
}

\author{
Ralf Jung and Tim Schwartz \\ Saarland University, Computer Science Department, Stuhlsatzenhausweg, \\ 66123 Saarbrücken, Germany \\ \{rjung, schwartz\}@es. uni-sb.de
}

\begin{abstract}
In this paper, we introduce an application for a discreet notification of mobile persons in a multi-user environment. In particular we use the current user position to provide a personalized email notification with non-speech audio cues embedded in aesthetic background music. The notification is done in a peripheral way to avoid distration of other people in the surrounding.
\end{abstract}

Keywords: Auditory Display, Ambient Soundscapes, Indoor Positioning.

\section{Introduction}

In our everyday life, people feel the need to be reachable in every situation. To guarantee this reachability, people make use of a variety of technologies, e.g. computers and mobile phones. A problem arises when a message or a phone call reaches the user and he must be notified about this event. In the context of a mobile phone this will usually be done by ring tones or vibration.

These types of notification have several disadvantages in situations like a business meeting. Not only is the lack of privacy a big disadvantage because attendees will know who received the message, but also the people`s attention in the room will be distracted from their current work. Even by switching off the ring tone and using the vibration alarm, the vibration could be audible (especially when the phone is lying on the desk) or the owner may not recognize the notification since the phone is in his jacket. The distraction problem raises from the fact that a notification signal is a "stand-alone" cue, that means the natural noise environment has a different sound type than the notification signal. The challenge is to increase the privacy by disguising the personal notification audio cue, which has the effect that nobody in the room can assign an appearing cue to the target person.

Our approach is to build up an artificial sound environment, the ambient soundscape, which is in our case a piece of non-speech easy listening music. This soundscape can be seen as an auditive envelope in which notification signals (in our case an instruments that fit into the composition) can be seamlessly integrated.

In our conference scenario, we assume a group of business men in a presentation who must be reachable the whole time or are waiting for an important email.

Registered users enter the conference room with their PDA that is equipped with an RFID reader card. The first user triggers the aforementioned ambient soundscape 
after his PDA receives the signal of one of the RFID tags that are mounted at the ceiling of the room. If an important email arrives (currently found out by keywordmatching of incoming emails), the current position of the user in the room will be determined by the PDA. These location coordinates will be send via wireless LAN to the system which determines the loudspeaker that is the nearest to the user. After knowing the position of the target person, his preselected favorite notification instrument (e.g. guitar) will be seamlessly mixed into the background music. To achieve this, a variety of musical constraints have to be considered whereas one of the most important constraint is the right point in time in which the notification instrument can be mixed into the soundscape without destroying the composition.

Unlike using the PDA loudspeakers for playing the notification signal, the use of the room speakers avoid the source detection of the notification signal. Not only the distraction can be avoided but also the privacy will be increased, because only the target person knows his personal notification instrument that he selected. Since the instrument fits into the composition other people will perceive the notification as part of the composition and not as a notification cue.

\section{Related Work}

Hudson and Smith designed a non-speech audio system that provides a preview of incoming emails by combining sample sounds [1]. The "audio glance" gives an overview of four important properties of a received message by coding information into the notification sound. First, the optional preamble sound is used for announcing messages that are classified as important. The sound of the main audio icon gives information about the message category, e.g. sender information in which the sample length represents the size of the mail body. For whom the mail is appointed to (single or group of users) is coded in the recipients icon and the finishing optional content flags announced mails where a keyword matching test for header or body is positive. The playback of the resulting sound could distract other people that are in the same room in which the notification takes place. Users can also receive their audio glance while they are away from their desk by holding up a color coded card in front of a camera. For multi-user environments, concurrently played samples could produce a confusing sound.

The Nomadic Radio [2] uses contextual audio cues on a wearable hands-free SoundBeam neckset for providing information. The scaleable audio interface remotes services and messages e.g. for email, news broadcasts and calendar events with wireless LAN and a telephony infrastructure. The interaction device is mounted on the shoulder of the user and is connected with a mini-portable PC that is also worn by the user. Messages are announced depending on the current user conversation context via speech and rendered spatial audio cues. Wearing such an additional special device that has only the function to receive auditive notifications could be hindering and reduce the acceptance. We decided to use standard PDAs for our notification system because the spread and popularity of PDAs increased in the last years. 


\section{The Ambient Email Notification Service}

Our claim to build a user centered notification service made a variety of demands on the architecture. Figure 1 gives a rough design overview of the four fundamental elements: Positioning System, eMail Server, Sound Repository and SAFIR (Spatial Audio Framework for Instrumented Rooms) that are used for the Ambient Email Notification service $(A e M N)$. In the graphical administration interface the user has the choice whether he wants to use the stationary or the mobile notification. The first one makes sense if the user stays at his desk. He can authenticate himself with his name and his password. The location of his personal desk and the email account login information are internally stored in an xml file on the AeMN server. The alternative is the mobile version where the user's PDA is already registered and used for finding out his current position. We assume that each user has his own PDA, so we don't need a manual authentication. In both cases the user has the possibility to enter a personal keyword for filtering incoming messages by their subject line. Incoming messages of registered users are periodically checked by email agents that run on the AeMN server.

After authentication, the user can select an ambient soundscape that ought to be played as the background sound. The system checks the sound repository for appropriate notification signals that can be integrated into the background soundscape as notification audio cues. After selecting his personal notification instrument, e.g. guitar or drums, the appropriate wav sound file will be retrieved from the sound repository and audio objects will be generated in the spatial audio system SAFIR [3].

AeMN recognizes when one or more registered users enter the room and automatically starts the selected backgound soundscape and the login process for checking the user accounts on the email server. The selected audio notification cues will be precached. If a user receives a new email that passed the filter successfully, the coordinates of the current user position that are computed by his PDA will be matched to the spatial audio system coordinates (listener position) and the notification cue will be seamlessly integrated into the soundscape. The loudspeaker that is nearest to the target person plays the notification cue with slightly increased volume to ease the perception. The notification can be stopped by pressing a button at a small user interface that is running on the PDA or at the administration interface on the desktop computer in case of the stationary version.

In the following we will give a more detailed overview of the structure and functionality of the sound repository and the positioning system.

\subsection{Ambient Notification with Personalized Audio Cues}

The main problem of traditional "stand alone" notification signals is the distraction of all present persons especially in multi-user environments. To introduce more privacy and confidentiality, we decided to integrate the notification signals with respect to the musical composition seamlessly into a background soundscape (see also [4]). The two musical components, namely the soundscapes and the notification instruments, were composed and recorded by ourselves. The compositions fulfill some perceptional constraints such as the auditive Gestalt laws [5], [6] and the consideration of the volume of the instruments that are well-known in musicology to influence the 


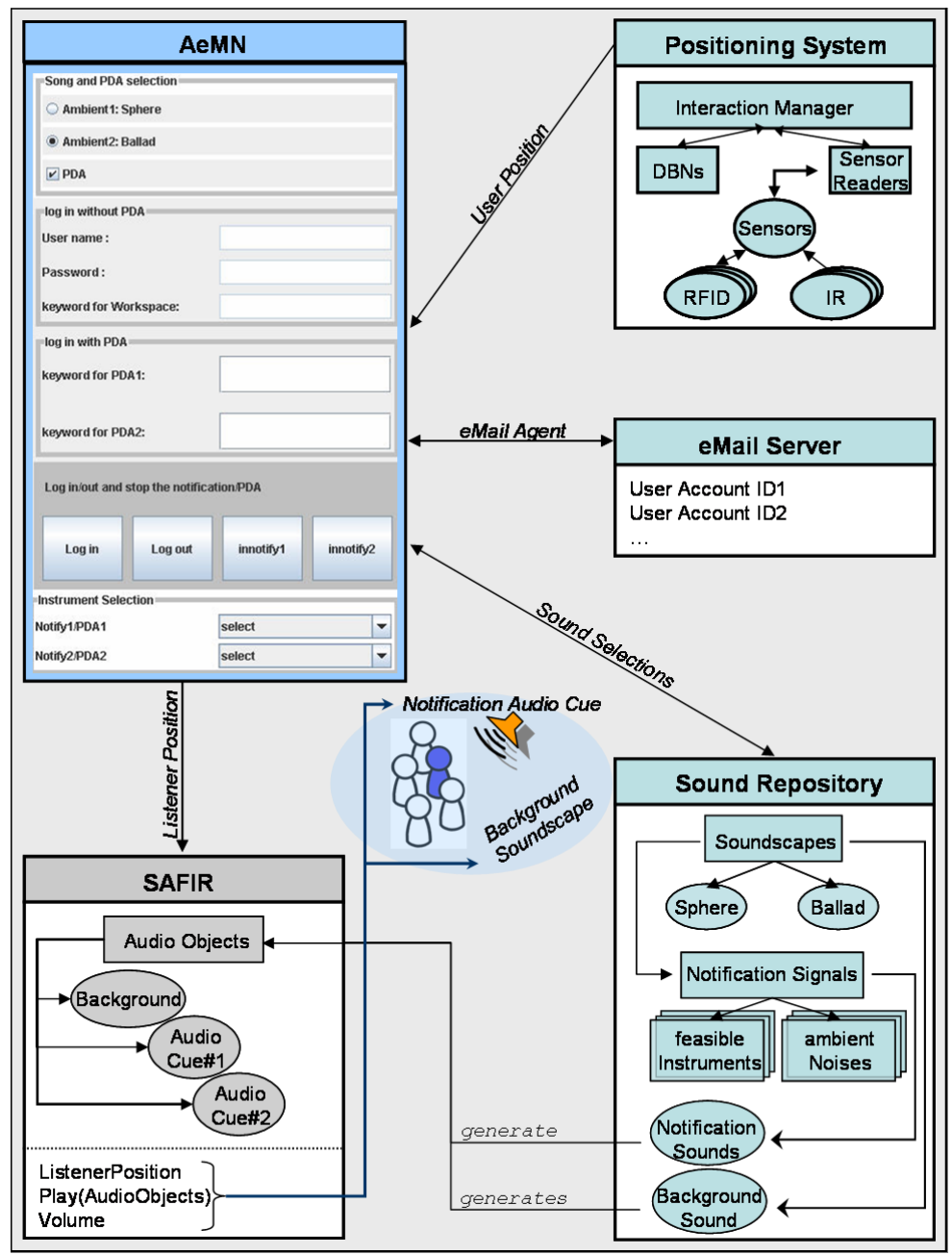

Fig. 1. Design Overview of the Audio Email Notification Architecture

perception process. The user has the possibility to choose a soundscape that matches his personal preferred music style and an instrument or ambient noise (natural sounds e.g. sea gull voices or flowing water) that he can easily recognize.

The notification cues can be mixed into the corresponding soundscape at certain points in time. We are obliged to this restriction to guarantee a fluent integration. Because of the fact that each user can select his personal instrument, other attendees will not be able to associate an instrument to a specific user even if they recognize the new cue. The personal instrument will seamlessly leave the soundscape if the user informs the system by pressing a button on his PDA or desktop GUI that he percepted the notification.

The effectiveness of the peripheral perception with our acoustical notification system was successfully tested in a user study with 25 persons where we especiallychecked 
whether the users percept the notification instruments and how much time they need to recognize the notification. More details about the study can be seen Section 4.

\subsection{Indoor Positioning for Location Awareness}

As stated above, the ambient notification system needs a way to find out about the current position of the users. The Global Positioning System (GPS) is well known for such tasks in outdoor environments, but due to physical constraints GPS normally is not working indoors. Finding ways to accomplish indoor localization is currently an interesting research topic, where the various ideas and technologies mainly differ in costs, precision and the used sensors and senders respectively.

For the ambient notification system, we use our own positioning system that is based on infrared beacons and active RFID tags. The corresponding sensors are the built in infrared port of the PDA and an RFID reader card that is attached to the PDA. It is important to notice that the senders (infrared beacons and RFID tags) are installed in the environment and the PDA with the sensors is worn by the user. The senders send out information about their own position, this information is read by the sensors and the user position is calculated on the PDA itself. The calculation is done with geo referenced Dynamic Bayesian Networks (geoDBNs), which cancel out false readings and combine the information of the different sensors (more details about geo referenced Dynamic Bayesian Networks, the accomplished sensor fusion and the positioning system itself can be read in [7], [8]).

Because the position is calculated on the personal device of the user (instead of a centralized server) his privacy is protected (only his device and the user himself know the current position). If the user wants to use special location aware applications, like the ambient notification service, he can choose to give away his position to these applications. With this mechanism the user can make a trade-off between privacy, benefit of and trust in an application.

\section{Design of Evaluation Study}

To test the viability of our ambient notification approach, we decided to compare it against a conventional acoustic alarm sound and conduct a study with the aim of answering the following two questions: For each kind of notification we want to know how often a notification is recognized (Efficiency) and with what delay the subjects react (Reaction time).

We recruited 25 participants (five women and twenty men) at ages from 20 to 35 years. Most of them had a background in either computer science or music.

\subsection{Setup of the Study}

The study was carried out in an instrumented room equipped with spatial audio hardware providing output through eight speakers mounted in a circular arrangement under the ceiling.

In this way, we were able to position the different parts of the soundscape and the specific notification instruments independently of each other. In addition to this, we prepared a computer with the test software. The study consisted of three parts with an overall duration of 30 minutes. 


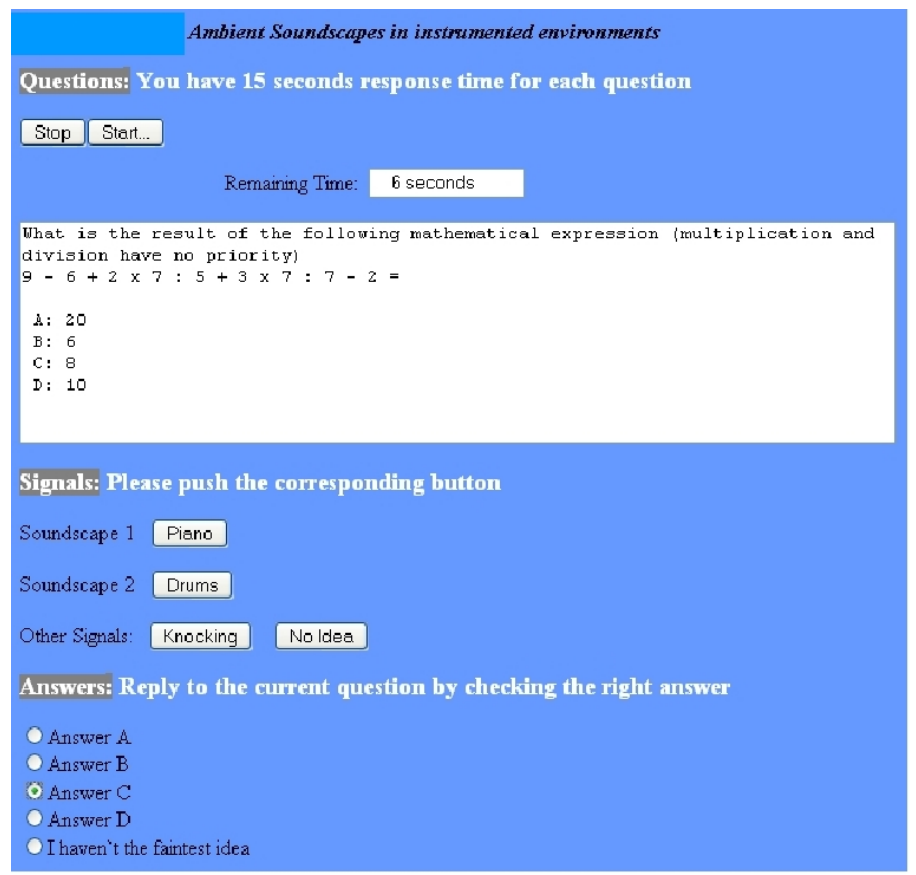

Fig. 2. Screenshot of the Computational Test

(1) Introduction and sound presentation (15 minutes)

In an explanatory text the subject was first introduced to the topic of the study and the test procedure and then given the opportunity to ask questions until we could ensure the tasks were fully understood. Subsequently, the subjects learned two personal notification signals and the corresponding soundscapes as well as the conventional alarm sound by repeated listening.

(2) Computer-based test (10 minutes)

The test environment included a question window, a signal button area and a radio button area for possible answers (see Figure 2). We prepared two recorded and prearranged soundscapes in which the notification instrument (learned by the subject in the introductory phase) and the conventional alarm sound appeared randomly. The task for the subject was to press the corresponding signal button after recognition of a notification signal as soon as possible. To prevent subjects from focusing on the background soundscape and to distract them from the auditive stimulus, they had to answer mathematical questions under time pressure. As a result of their increased cognitive load, the subjects perceived the audio signals in a rather peripheral way. In ambient soundscape ASO1, the piano was the relevant notification instrument. In the second soundscape $\mathrm{ASO2}$ we chose the drums as the audio cue. In contrast to the melody-dominated piano, the drums in AS02 are more rhythmically oriented. As a salient but natural traditional acoustic alarm signal we added a knocking sound randomly to both soundscapes. The volume of salient audio cues is important for the recognition process and we took great care to play the 
knocking sound at the same volume level as the notification instruments, but while these were part of the composition and matched its overall rhythmic and melodic structure, the knocking stood outside of the overall composition. The two soundscapes were played in a row. Users were told to push the corresponding signal button as soon as possible when they perceived an audio cue. The timing of the audio cues and the signal buttons were recorded to measure reaction times. The knocking sound and each notification instrument appeared five times for each subject.

(3) Questionnaire (5 minutes)

After the test, subjects were given a questionnaire of three pages with different styles of questions. The results are personal opinions and can be influenced by many factors. Thus we only used the questionnaire for retrieving additional information, not for deriving quantitative data.

\subsection{Results}

Our main interest was whether the proposed notification system works efficiently (and potentially more peripheral than traditional audio notification systems). We also looked at the reaction time and some additional information extracted from the questionnaire.

\subsubsection{Notification Efficiency}

Over the course of all 25 subjects, there were 125 piano and 125 drum cues. Of these, 98 piano and 109 drum cues were recognized and identified by the subjects. The standard deviation of the rate for the drum signal with a value of 0,162 is lower than the piano value $(0.244)$ and clearly lower than the knocking deviation $(0,286)$.
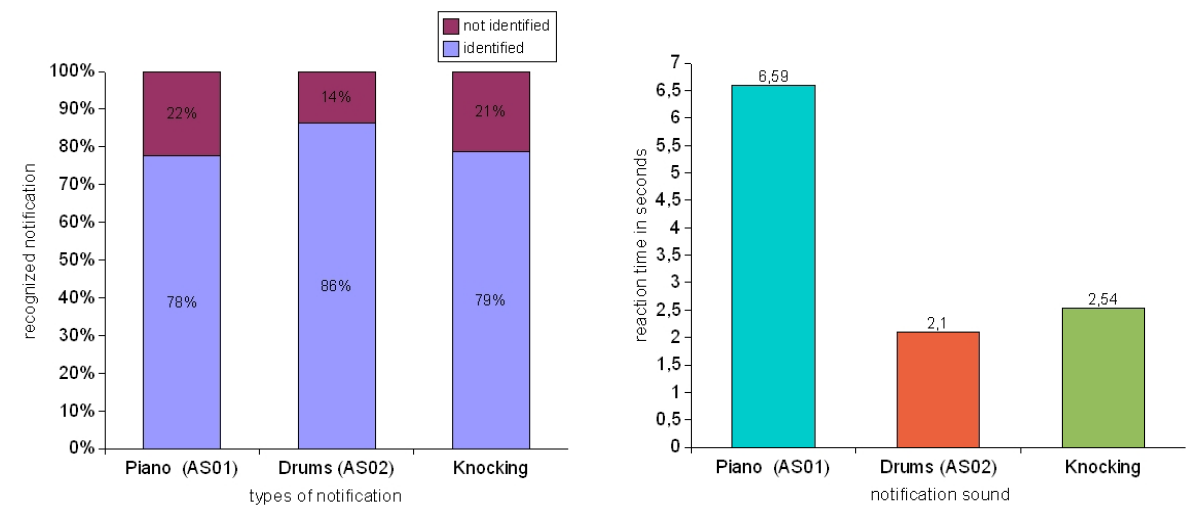

Fig. 3. Overall Notification Efficiency (left) and Overall Reaction Time (right)

This suggests that the more rhythmical drums are easier to identify than the a melodical instrument like the piano. Compared to the conventional alarm signal (knocking), the efficiency is surprisingly high (Fig. 2). Especially the drum notification in AS02 surpassed the knocking sound by seven percent and proved to be the most efficient of the three notification types. 


\subsubsection{Reaction Time}

We were also interested in the delay between the notification appearance and the act of pressing the button (Fig. 3). Subjects had to perceive the audio signal, identify it and press the corresponding signal button on the screen. We found out that the average reaction time for piano notifications was higher on average $(6,59$ seconds) than the reaction time for drum $(2,1$ seconds) and knocking notifications $(2,54$ seconds).

We observed all subjects during the test and took notes whether they first answered the current question or first pressed the signal button. We found out that there seemed to be two types of perception:

- Immediate Perception. The subject recognized the audio signal in the first five seconds after its appearance. The audio cue was focused immediately after the stimulus perception.

- Memorized Perception. The test person pressed the button after the audio signal had already disa.ppeared. The reason for this phenomenon was an effect often described with the words "I think I have heard a signal". In general, subjects which had stated in the questionnaire that they were difficult to distract form their current work had a longer reaction time or missed notifications completely.

\section{Conclusions and Future Work}

We introduced an ambient notification service that works with personalized audio cues and that adapts to the position of the user with the help of his PDA. Occuring events, in our case an incoming email where the subject line matches a preselected

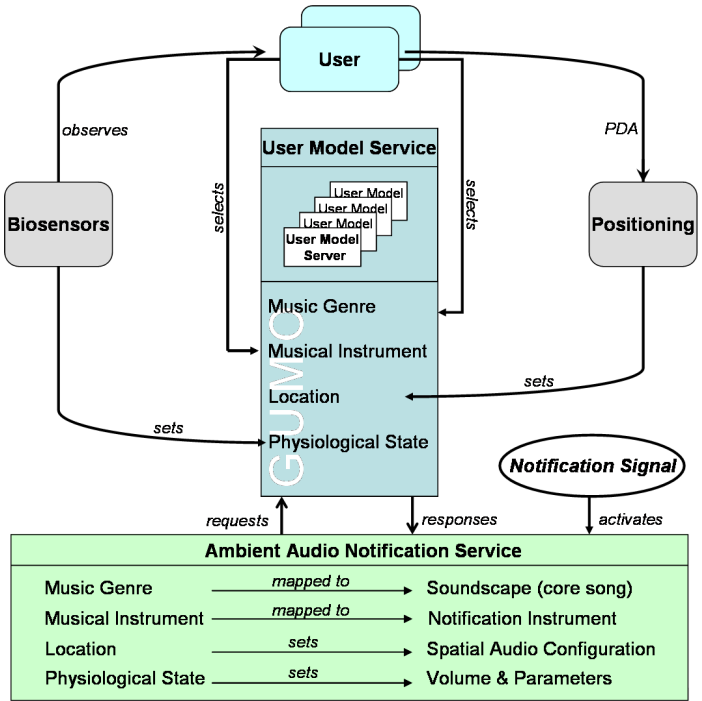

Fig. 4. User Model Integration for Ambient Audio Notification keyword, can be announced by enhancing a background soundscape with a personal cue that is played near the user's current position with increased volume. This type of unobtrusive notification gives us the chance to follow a low-level privacy approach. Areas of applications are shops where employees can receive information (e.g. a cashier is needed in the point of sale area) and at the same time the background soundscape would have a comfortable effect on the customers.

The future work includes the connection to the General User Model Ontology GUMO [9], [10] to provide a more adaptive and flexible notification service for 
instrumented rooms (Figure 4). The enhanced personalization features will include the position of the user, his personal music style and favorite instruments and his physical state that we will try to find out with biosensors connected to the user [11]. The individual settings can then be accessed via http requests when the user enters a room.

\section{References}

1. Hudson, S.E., Smith, I.: Electronic Mail Previews Using Non-Speech Audio. In: CHI '96: Conference companion on Human factors in computing systems, New York, NY, USA, pp. 237-238. ACM Press, New York (2006)

2. Sawhney, N., Schmandt, C.: Nomadic Radio: Scaleable and Contextual Notification for Wearable Audio Messaging. In: CHI '99: Proceedings of the SIGCHI conference on Human factors in computing systems, pp. 96-103. ACM Press, New York, NY, USA (1999)

3. Schmitz, M., Butz, A.: Safir: Low-Cost Spatial Audio for Instrumented Environments. In: Proceedings of the 2nd International Conference on Intelligent Environments, Athens, Greece (2006)

4. Butz, A., Jung, R.: Seamless User Notification in Ambient Soundscapes. In: IUI '05: Proceedings of the 10th international conference on Intelligent user interfaces, pp. 320 322. ACM Press, New York, NY, USA (2005)

5. Camurri, A., Leman, M.: Gestalt-Based Composition and Performance in Multimodal Environments. In: Joint International Conference on Cognitive and Systematic Musicology, pp. 495-508 (1996)

6. Reybrouck, M.: Gestalt Concepts and Music: Limitations and Possibilities. In: Joint International Conference on Cognitive and Systematic Musicology, Brugge, Belgium, pp. 57-69 (1997)

7. Brandherm, B., Schwartz, T.: Geo Referenced Dynamic Bayesian Networks for User Positioning on Mobile Systems. In: Strang, T., Linnhoff-Popien, C. (eds.) LoCA 2005. LNCS, vol. 3479, pp. 223-234. Springer, Berlin, Heidelberg (2005)

8. Schwartz, T., Brandherm, B., Heckmann, D.: Calculation of the User-Direction in an Always Best Positioned Mobile Localization System. In: Proceedings of the International Workshop on Artificial Intelligence in Mobile Systems (AIMS), Salzburg, Austria (2005)

9. Heckmann, D., Schwartz, T., Brandherm, B., Kroener, A.: Decentralized User Modeling with UserML and GUMO. In: Proceedings of the Workshop on Decentralized, Agent Based and Social Approaches to User Modelling, (DASUM 2005), Edinburgh, Scotland, pp. 61-65 (2005)

10. Jung, R., Heckmann, D.: Ambient Audio Notification with Personalized Music. In: Proceedings of Workshop on Ubiquitous User Modeling (UbiqUM'06), Riva del Garda, Italy, pp. 16-18 (2006)

11. Brandherm, B., Schultheis, H., von Wilamowitz-Moellendorff, M., Schwartz, T., Schmitz, M.: Using Physiological Signals in a User-Adaptive Personal Assistant. In: Proceedings of the 11th International Conference on Human-Computer Interaction (HCII-2005), Las Vegas, Nevada, USA (2005) 